\title{
Clustering of Cell Surface Laminin Enhances Its Association with the Cytoskeleton
}

\author{
ROBERT L. CODY and MAX S. WICHA \\ Simpson Memorial Research Institute, Division of Hematology and Oncology, Department of \\ Internal Medicine, University of Michigan, Ann Arbor, MI 48109, USA
}

\begin{abstract}
In order to provide evidence for an association of cell surface laminin with the cytoskeleton, we have examined the detergent extractability of cell surface laminin on murine fibrosarcoma cells. We utilized indirect immunofluorescence with affinity-purified antilaminin antibodies to determine the distribution, mobility and detergent extractability of laminin bound to the cell surface. We demonstrate that antibody induces clustering of cell surface laminin rendering it resistant to detergent extraction.

At low receptor occupancy, approx. $80 \%$ of cell surface laminin is detergent-extractable. If cell surface laminin is induced to cluster with anti-laminin antibody, $\mathbf{I B}_{4}$ isolectin from Bandeiraea simplicifolia or by high receptor occupancy, then it is rendered resistant to detergent extraction. This process is temperature-sensitive and inhibited by cytochala$\sin \mathrm{D}(\mathrm{CD})$. On the basis of these findings, we propose a model in which laminin anchored in the basement membrane in vivo affects the cellular cytoskeleton by facilitating the clustering of cell surface transmembrane laminin receptors which are able to interact with cellular actin. (c) 1986 Academic Press, Inc.
\end{abstract}

It is now clear that components in the extracellular matrix have important effects on cellular migration, growth and differentiation $[1,2]$. Although the mechanisms by which extracellular matrix components mediate these effects are largely unknown, there is now evidence that cells may interact with extracellular matrix via specific cell surface receptors. We have recently described the isolation of a cell surface protein of $\mathrm{M}_{r}$ approx. $70 \mathrm{kD}$ from murine fibrosarcoma cells which binds the basement membrane glycoprotein laminin with specificity and high affinity (kD $2 \mathrm{nM}$ ) [3]. Similar laminin receptor proteins have now been described in a variety of cell types, including normal and malignant mammary cells, muscle cells, hepatocytes and inflammatory cells $[4,6]$. In addition, receptors for fibronectin and collagen have also recently been described [7-10].

It has been suggested that the effects of extracellular matrix components on cellular behaviour may be mediated through the cytoskeleton. Sugrue \& Hay [11] have demonstrated that a variety of extracellular matrix components, including soluble laminin, can cause a reorganization of the cellular cytoskeleton. They postulated that these effects might be mediated by specific cell surface receptors capable of interacting with both extracellular matrix components and the cytoskeleton. We have recently found that the $70 \mathrm{kD}$ laminin receptor protein purified from murine fibrosarcoma cells is also capablc of binding to cellular cytoskeletal actin in vitro [12]. This binding was demonstrated both by co-sedimentation of the receptor with actin and the binding of the receptor to actin immobilized on 

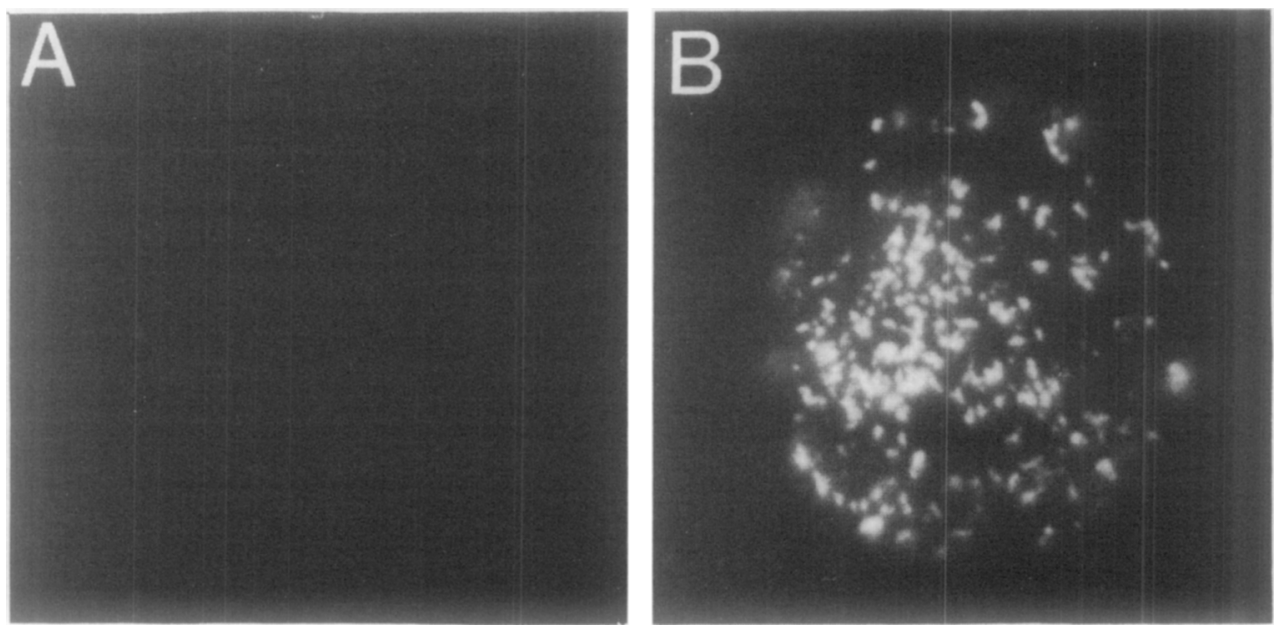

Fig. 1. Distribution of cell surface laminin by immunofluorescence. Np cells were prepared and indirect immunofluorescence performed as described in Methods. ( $A$ ) The experiment was performed at $4^{\circ} \mathrm{C}$. Cells were treated sequentially with laminin, anti-laminin, rhodamine-conjugated second antibody, followed by extraction with Triton $\mathrm{X}-100$. $(B)$ The experiment was performed at $22^{\circ} \mathrm{C}$, otherwise cell treatment was the same as in $(A)$. Not shown are control experiments in which cells were treated as in $(A)$ and $(B)$ with the elimination of either laminin or first antibody (anti-laminin). No fluorescence was noted in either control.

nitrocellulose filters. The receptor reduced the viscosity of actin filaments and caused the bundling of parallel filaments.

Based on the ability of this receptor to bind both extracellular laminin and intracellular actin, we named the protein 'connectin' [12]. We postulate that connectin is a transmembrane protein capable of mediating the interaction of the cellular cytoskeleton and the extracellular matrix. In order to provide evidence for a functional link between cell surface laminin and the cytoskeleton, we have now studied the ability of detergent to extract laminin bound to the surface of murine fibrosarcoma cells. We report that laminin can be induced to cluster on the surface of cells using a variety of agents and that clustered cell surface laminin is resistant to detergent extraction. This process is temperature-sensitive and is inhibited by cytochalasin $\mathrm{D}(\mathrm{CD})$ which blocks actin polymerization. Based on these findings, we propose a model in which the configuration of laminin in the extracellular matrix affects the organization of actin in the cellular cytoskeleton through the clustering of transmembrane laminin receptors.

\section{MATERIALS AND METHODS}

\section{Cells}

$\mathrm{Np}$ cells derived from a methylcholanthrene-induced murine fibrosarcoma were cultured as previously described [13]. These cells display cell surface laminin receptors but have no detectable endogenous cell surface laminin [3]. 


\section{Reagents}

Laminin. Laminin was prepared from the EHS (Engelbreth-Holm-Swarm) tumour maintained in C57/BL6 mice [14]. Purity was verified by gel electrophoresis. Laminin-coated dishes were prepared by drying laminin at $2 \mu \mathrm{g} / \mathrm{cm}^{2}$ on tissue culture chamber slides (Miles Scientific, Naperville, Ill.). Laminin was iodinated to a specific activity of $4 \mu \mathrm{Ci} / \mu \mathrm{g}$ using Enzymobeads (Bio-Rad, Richmond, Calif.). Polyclonal anti-laminin antibody, raised in rabbits and, purified by affinity chromatography has previously been characterized [15]. $\mathrm{IB}_{4}$ isolectin from Bandeiraea simplicifolia was obtained from Sigma (St. Louis, Mo.).

\section{Immunofluorescence}

Cells were harvested using $3 \mathrm{mM}$ EDTA in Earle's Balanced salt solution (EBSS). $3 \times 10^{6}$ cells were incubated with $20 \mu \mathrm{g}$ laminin in $0.5 \mathrm{ml}$ EBSS for $30 \mathrm{~min}$. All procedures were performed at $22^{\circ} \mathrm{C}$ unless otherwise noted. Following washing $\times 2$ in EBSS to remove unbound laminin, cells were incubated with a 1:100 dilution of anti-laminin antibody for $20 \mathrm{~min}$, washed and then incubated with a $1: 100$ dilution of rhodamine-conjugated goat anti-rabbit antibody for $20 \mathrm{~min}$. Following washing, cells were mounted in $20 \%$ glycerol in EBSS and examined for fluorescence [15]. Where indicated, cells were extracted with $1 \%$ Triton X-100 (Sigma) for $10 \mathrm{~min}$. In order to detect F-actin, rhodamine-conjugated phalloidin was utilized. Cells were allowed to attach to tissue culture or laminin-coated slides, fixed with $3 \%$ formaldehyde for $10 \mathrm{~min}$ and extracted with $1 \%$ Triton X-100 for $10 \mathrm{~min}$. One aliquot of cells was preincubated with $5 \mu \mathrm{M}$ cytochalasin D (Sigma) for $1 \mathrm{~h}$ prior to harvesting.

\section{Quantitation of Bound Cell Surface Laminin}

$3 \times 10^{6}$ cells were incubated with $2 \times 10^{6} \mathrm{cpm}$ [ $\left.{ }^{125} \mathrm{I}\right]$ laminin $(4 \mu \mathrm{Ci} / \mu \mathrm{g})$ for $30 \mathrm{~min}$. All procedures were performed at $22^{\circ} \mathrm{C}$. To quantitate laminin bound to the detergent resistant cytoskeleton, cells were solubilized with $1 \%$ Triton X-100 for $10 \mathrm{~min}$. We have previously demonstrated that this concentration of detergent has no effect on the binding of laminin to purified laminin receptor [3]. Control cells were treated with EBSS instead of detergent. Unbound laminin was separated from laminin bound to the cell surface or cytoskeleton by sedimentation through a Percoll cushion (Sigma). $150 \mu \mathrm{l}$ of extract was layered on $150 \mu \mathrm{l}$ of $15 \%$ Percoll in EBSS in a $400 \mu \mathrm{l}$ conical tube (Beckman, Palo Alto, Calif.) and centrifuged at $12000 \mathrm{~g}$ for $10 \mathrm{~min}$. Whole cells, cytoskeletal components and associated proteins sediment through the Percoll, while unbound $\left[{ }^{125}\right.$ I]laminin does not.

Following sedimentation the tubes were clamped, cut above the pellet, and [ ${ }^{125}$ I]laminin in the pellet assessed by gamma counting. Data are expressed as percent laminin associated with the cytoskeleton calculated as:

cpm pellet in presence of detergent $\times 100$.

$\mathrm{cpm}$ pellet in absence of detergent

The effects of anti-laminin antibody and $\mathbf{I B}_{4}$ isolectin from Bandeiraea simplicifolia on laminin association with the cytoskeleton was determined using detergent extraction and Percoll sedimentation as described above. $3 \times 10^{6}$ Cells were incubated with [ ${ }^{125}$ I]laminin, washed and then incubated with either a 1:100 dilution of anti-laminin antibody or varying concentrations of $\mathrm{IB}_{4}$ isolectin, washed three times and then extracted with $1 \%$ Triton X-100. Cytoskeletal associated $\left.{ }^{125} I\right]$ laminin was determined by sedimentation through Percoll as desribed above. Duplicate samples not treated with detergent were used to determine total laminin bound to the cell surface. Where indicated, cells were cultured in the presence of $5 \mu \mathrm{M} \mathrm{CD}$ for $1 \mathrm{~h}$ prior to harvesting. This $\mathrm{CD}$ pretreatment had no effect on laminin binding to the cell surface. In figs 3 and 4 , the data shown represent the mean of triplicate values with $\mathrm{SE}<10 \%$ of mean.

\section{RESULTS}

\section{Distribution and Detergent Extractibility of Cell Surface Laminin}

We used immunofluorescence with affinity-purified anti-laminin antibodies to determine the distribution and mobility of laminin on the cell surface. When 

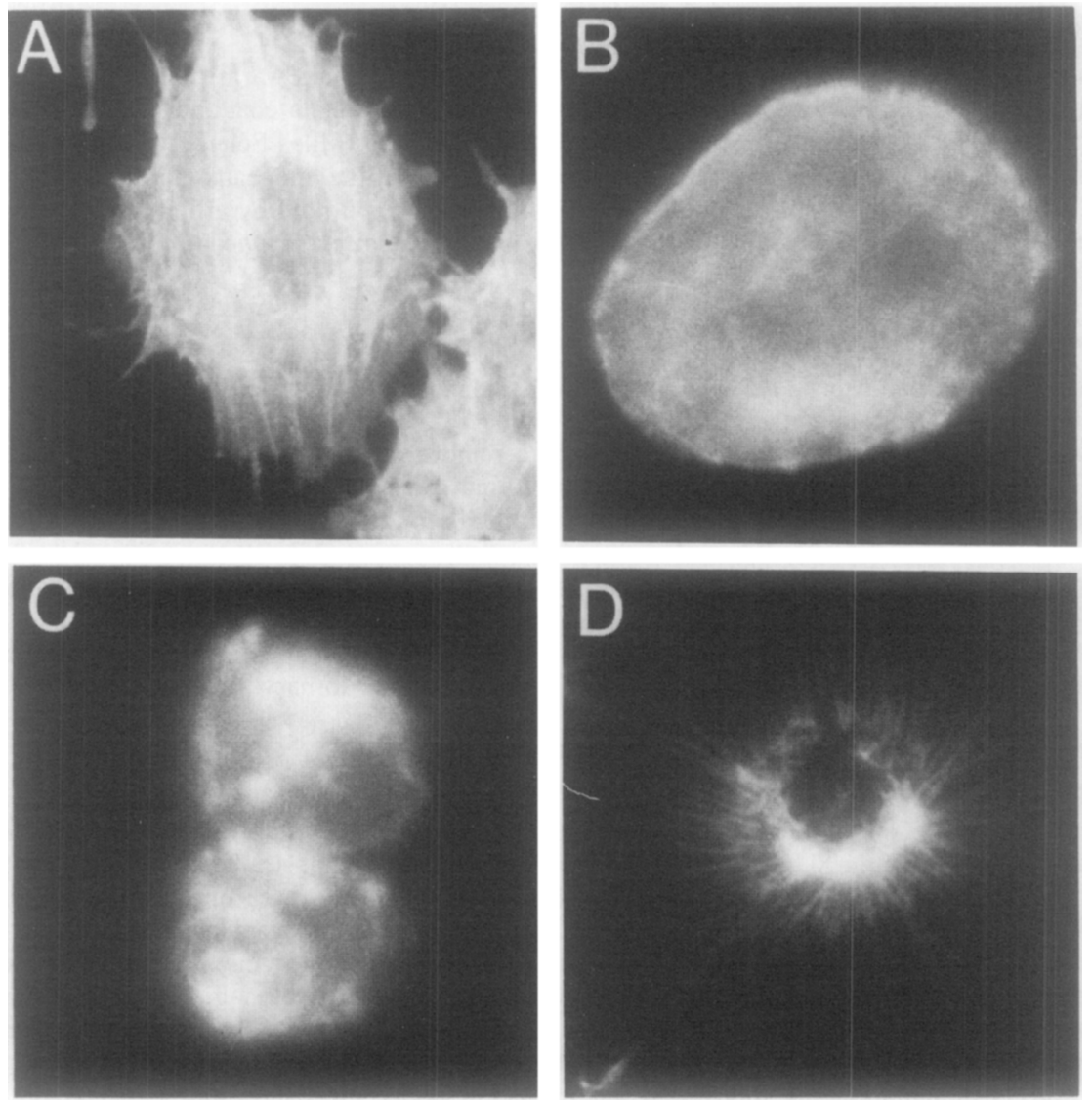

Fig. 2. Cellular actin distribution. Np cells were allowed to attach to substratum for $60 \mathrm{~min}$ at $37^{\circ} \mathrm{C}$, fixed with formaldehyde, then detergent-solubilized. They were then treated with rhodamine-conjugated phalloidin and examined by fluorescence microscopy to detect F-actin. (A) Laminin-coated slide. Note actin organization. $(B)$ Uncoated slide. $(C)$ Cells pretreated with $5 \mu \mathrm{M} C D$ before attachment to laminin-coated slide. (D) Unfixed cells on laminin-coated slides were detergentsolubilized and actin-localized as above. Note attachment of actin filaments to substratum.

laminin-coated cells were incubated at $22^{\circ} \mathrm{C}$ with affinity-purified anti-laminin antibody and rhodamine-conjugated second antibody, clusters resistant to detergent extraction were seen (fig. $1 \mathrm{~B}$ ). If these experiments were performed at $4^{\circ} \mathrm{C}$ to prevent clustering, then the fluorescence was removed with detergent extraction (fig. 1A). These experiments suggest that laminin clustering by antibody is temperature-dependent. Furthermore, the resistance of clustered cell surface laminin to detergent extraction suggest an association with the cytoskeleton.

\section{Distribution of Cellular Actin}

The above experiments suggested the association of clusters of cell surface laminin with the cytoskeleton. In the basement membrane in vivo laminin molecules are immobilized by binding to other basement membrane components [16]. 
Table 1. Effect of anti-laminin antibody on laminin-cytoskeleton association ${ }^{a}$

\begin{tabular}{lll}
\hline $\begin{array}{l}\text { Detergent } \\
\text { alone }\end{array}$ & $\begin{array}{l}\text { Anti-laminin antibody } \\
\text { prior to detergent }\end{array}$ & $\begin{array}{l}\text { Detergent prior to anti- } \\
\text { laminin antibody }\end{array}$ \\
\hline $18 \pm 1$ & $66 \pm 6$ & $24 \pm 2$ \\
\hline
\end{tabular}

a Values shown are percentage detergent-resistant laminin $\pm \mathrm{SE}$ determined as described in Methods.

Cells were treated with trace amounts of $\left[{ }^{125}\right.$ I]laminin ( $1 \mathrm{ng}$ bound $/ 6 \times 10^{5}$ cells). One aliquot was treated with anti-laminin antibody (1:100 dilution), free antibody removed, and cells were then treated with Triton X-100. Cytoskeletal associated laminin was determined by sedimentation through Percoll as described in Methods. Another aliquot was treated with Triton X-100 prior to the antilaminin.

We therefore determined the effect of a laminin substratum on cellular actin organization using rhodamine-conjugated phalloidin to detect F-actin. When Np cells were incubated on laminin-coated slides, they attached, spread, and rapidly developed an organized network of actin filaments (fig. $2 A$ ). Cell spreading and actin organization did not occur during this time period on control slides that were not laminin-coated (fig. $2 B$ ). Actin organization in cells on laminin-coated slides was disrupted by $C D$ (fig. $2 C$ ). When unfixed cells on laminin substrata were extracted with detergent prior to treatment with rhodamine phalloidin, a dense network of actin filaments remained associated with the substratum (fig. 2D).

\section{Quantitation of Cell Surface Laminin-Cytoskeleton Association}

In order to quantitate the association of cell surface laminin with the cytoskeleton, $\mathrm{Np}$ cells were incubated with [ $\left.{ }^{125} \mathrm{I}\right] \mathrm{laminin}$, and then extracted with Triton X-100 detergent. Cell membrane components not associated with the cytoskeleton are removed by this procedure $[16,17]$. As shown in table 1 , when trace amounts of $\left[{ }^{125} \mathrm{I}\right] \mathrm{laminin}$ are bound to $\mathrm{Np}$ cells, $82 \%$ of the bound laminin is extractable, indicating that under these conditions $18 \%$ of cell surface laminin is associated with the detergent-resistant cytoskeleton.

Table 2. Effect of temperature on laminin-cytoskeleton association ${ }^{a}$

\begin{tabular}{rll}
\hline & $\begin{array}{l}\text { Low receptor } \\
\text { occupancy }\end{array}$ & $\begin{array}{l}\text { Higher receptor } \\
\text { occupancy }\end{array}$ \\
\hline $22^{\circ} \mathrm{C}$ & $22 \pm 1$ & $48 \pm 1$ \\
$4^{\circ} \mathrm{C}$ & $25 \pm 2$ & $28 \pm 1$ \\
\hline
\end{tabular}

${ }^{a}$ Values shown are percent detergent resistant $\mathrm{cpm} \pm \mathrm{SE}$. Cells were incubated with trace amounts of [ $\left.{ }^{125} \mathrm{I}\right]$ laminin. 'Low' receptor occupancy represents $1.85 \mathrm{ng}$ laminin bound per $10^{6}$ cells $(1.4 \%$ of receptors occupied), and 'higher' receptor occupancy represents $15.8 \mathrm{ng}$ laminin was bound per $10^{6}$ cells (19\% of receptors occupied). Laminin-cytoskeleton association was determined by detergent extraction followed by sedimentation through Percoll as in table 1. 

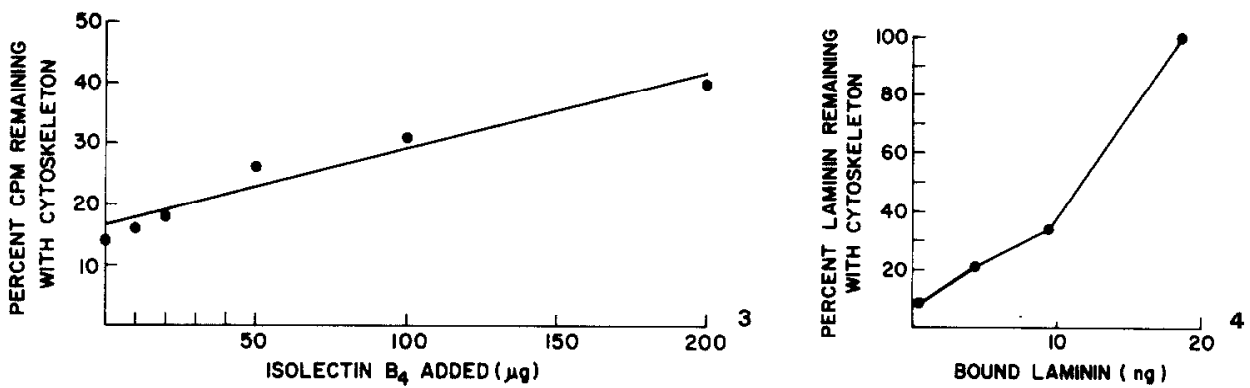

Fig. 3. Effect of $\mathrm{IB}_{4}$ isolectin on laminin cytoskeletal association. Cells were incubated with trace amounts of $\left[{ }^{125} \mathrm{I}\right] \mathrm{laminin}\left(1 \mathrm{ng}\right.$ bound $/ 6 \times 10^{5}$ cells). They were then incubated with increasing concentrations of $\mathrm{IB}_{4}$ isolectin from Bandeiraea simplicifolia and the percentage laminin associated with the cytoskeleton was determined as in table 1.

Fig. 4. Effect of increasing surface-bound laminin on its association with the cytoskeleton. $3 \times 10^{5}$ cells were incubated with laminin of varying specific activities. For each point, one set of cells were detergent-treated. Total laminin, bound in the absence of detergent, was calculated based on $\mathrm{cpm}$ bound and laminin-specific activity. A duplicate set of tubes was detergent-extracted and the percentage of cell surface laminin associated with the cytoskeleton was calculated as described in Methods.

Effect of cross-linking agents on laminin-cytoskeleton association. The immunofluorescence studies described above suggested that a multivalent ligand such as anti-laminin antibody could produce clustering of cell surface laminin rendering it resistant to detergent extraction. This was confirmed and quantitated using surface-bound [ ${ }^{125}$ I]laminin. Anti-laminin antibody addition prior to detergent extraction increased the detergent-resistant fraction from $18 \%$ to $66 \%$. This effect was not seen if the cells were treated with detergent prior to antibody addition (table 1). These observations are consistent with the hypothesis that anti-laminin antibody promotes clustering of cell surface laminin promoting its association with the cytoskeleton.

$B_{4}-$ Isolectin from Bandeiraea simplicifolia. The $\mathrm{B}_{4}$ isolectin from Bandeiraea simplicifolia is a multivalent lectin which is capable of binding to the terminal $\alpha$ D-galactose groups of murine laminin [18]. We determined the effect of this lectin

Table 3. CD effect of cytochalasin D on laminin cytoskeletal association ${ }^{a}$

\begin{tabular}{rll}
\hline & $\begin{array}{l}\text { Low receptor } \\
\text { occupancy } \\
(-)\end{array}$ & $\begin{array}{l}\text { High receptor } \\
\text { occupancy } \\
(+)\end{array}$ \\
\hline $\mathrm{CD} \mathrm{(-)}$ & $16 \pm 1$ & $89 \pm 2$ \\
$(+)$ & $12 \pm 1$ & $35 \pm 2$
\end{tabular}

${ }^{a}$ Values shown are percentage detergent resistant laminin $\pm \mathrm{SE}$ as in table 2 .

Cells with low and high occupancy of laminin receptors were incubated in the presence or absence of $\mathrm{CD}(5 \mu \mathrm{M})$ for $60 \mathrm{~min}$. Laminin associated with cytoskeleton was determined as in table 1 . Low occupancy represents $1.6 \mathrm{ng}$ laminin bound $/ 10^{6}$ cells $(1.9 \%$ laminin receptors occupied). High occupancy represents $74.0 \mathrm{ng}$ laminin bound $/ 10^{6}$ cells $(90 \%$ of laminin receptors occupied). 
on the association of the cell surface laminin with the cytoskeleton. As determined by resistance to the detergent extraction, $\mathrm{IB}_{4}$ increased the association of cell surtace laminin with the cytoskeleton in a dose-dependent manner (fig. 3).

Effect of increasing cell surface laminin on its association with the cytoskeleton. The above experiments were performed with trace amounts of laminin (1 ng bound $/ 10^{6}$ cells). If one assumes that $\mathrm{Np}$ cells have $5 \times 10^{4}$ laminin receptors per cell, as we have previously determined [3], and that one receptor binds one laminin molecule, then the [ ${ }^{125}$ I]laminin used in the above experiments occupy approx. $1 \%$ of the available receptors. We examined the effect of increasing amounts of cell surface laminin on its association with the cytoskeleton. This was done by increasing the amount of laminin added to the cells (varying the specific activity). As can be seen in fig. 4, the percentage of cell surface laminin associated with the cytoskeleton increases as the amount of cell surface laminin bound increases. When $>90 \%$ of the cell surface laminin receptors are occupied, they are totally non-extractable with detergent (fig. 4).

\section{Inhibition of Laminin-Cytoskeleton Association}

Temperature. In a variety of systems, clustering of cell surface receptors is a temperature-dependent phenomenon [17]. We therefore examined the effect of temperature on the association of laminin with the cytoskeleton. As shown in table 2, the potentiation of laminin-cytoskeleton association due to high receptor occupancy was not seen at $4^{\circ} \mathrm{C}$. This is consistent with the qualitative observations in fig. 1. Thus, clustering of cell surface laminin is potentiated by high receptor occupancy and is temperature-dependent.

Cytochalasin D. We examined the effects of cytochalasin D (CD), which inhibits actin polymerization, on laminin association with the cytoskeleton. Consistent with results seen in fig. 4 , increasing amounts of cell surface laminin increased the percentage associated with the cytoskeleton from $16 \%$ to $89 \%$ (table 3). In contrast, in CD-treated cells, the percentage of cell surface laminin associated with the cytoskeleton under these conditions increased only from 12 to $35 \%$.

\section{DISCUSSION}

In this report we provide evidence for an association of cell surface laminin with the cytoskeleton. This is demonstrated by the resistance of cell surface laminin to detcrgent extraction. Furthermore, we demonstrate qualitatively by immunofluorescence and quantitatively by [ ${ }^{125}$ I]laminin binding that the clustering of cell surface laminin by cross-linking agents increases its association with the cytoskeleton. This process is temperature-sensitive and is inhibited by $C D$. On the basis of these experiments, we propose a model in which laminin immobilized in the basement membrane in vivo is able to organize actin in the cytoskeleton. This would occur through a clustering of cell surface laminin receptors which we 
A

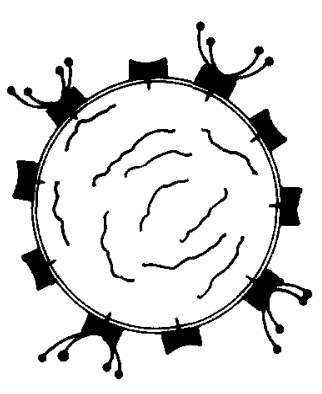

B

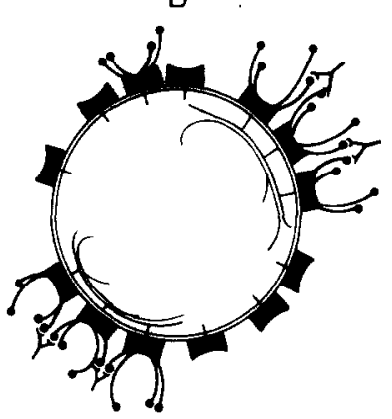

$\mathrm{C}$

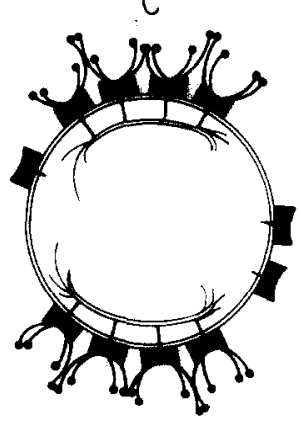

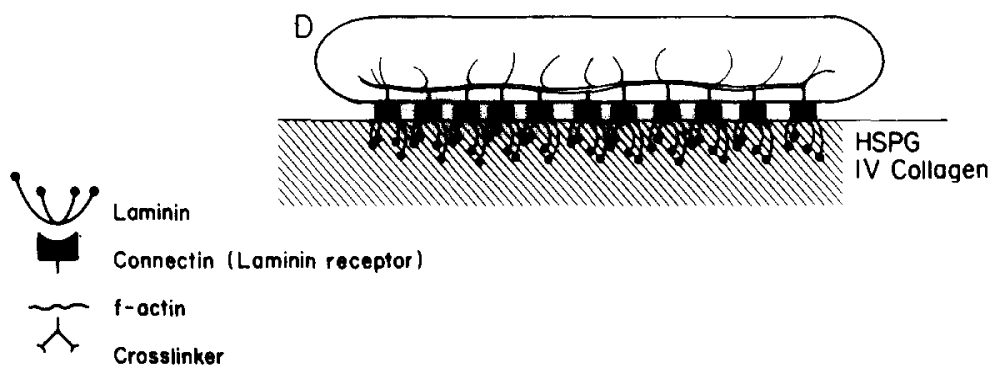

Fig. S. Schematic representation of laminin-connectin-actin association. (A) Laminin receptor occupancy low. Connectin distributed throughout the cell membrane of cells in suspension. The connectin is not clustered; actin is not bound to connectin. $(B)$ Cell surface laminin cross-linked with $\mathrm{IB}_{4}$ isolectin or anti-laminin antibody. Actin bound to clustered connectin. (C) Laminin receptor occupancy high. Side-to-side association of laminin molecules [22] faciliates clustering of connectin. Actin bound to clustered connectin. (D) Cells attached to basement membrane. Laminin anchored in the basement membranes causes clustering of connectin on the basal cell surface. Actin is bound to the clustered connectin establishing cytoskeletal organization, and cell polarity.

postulate are transmembrane proteins capable of binding to the sides of actin filaments. This model is depicted in fig. 5 .

In previous studies, we isolated a cell surface laminin receptor protein from murine fibrosarcoma cells. This $68-70 \mathrm{kD}$ protein binds to laminin with specificity and high affinity [3]. Similar laminin receptor proteins have now been isolated from a variety of cell types [4, 5]. We have also demonstrated previously that laminin receptor protein purified from murine fibrosarcoma cells is capable of binding to the sides of actin filaments in vitro [12]. Based on the ability of this laminin receptor protein to bind extracellular laminin and intracellular actin, we have named the protein 'connectin'. However, the observation that connectin and actin can interact in vitro does not necessarily indicate that a similar process occurs in the cell. The resistance of clustered cell surface laminin to detergent extraction provides further evidence for a linkage of cell surface laminin to the cytoskeleton in vivo.

There are several mechanisms by which the binding of laminin to its receptor 'connectin' could influence its subsequent interaction with actin. One mechanism postulates a conformational change in the connectin molecule induced by laminin 
which subsequently affects its affinity for actin. An alternative possibility which does not require a conformational change, is that the clustering of laminin on the cell surface results in the clustering of connectin. Since we postulate that connectin is a transmembrane protein capable of binding to the sides of actin filaments, then the clustering of connectin would bring together multiple actin-binding domains on the inner surface of the plasma membrane. This clustering would lead to an increased apparent affinity of connectin for actin compared with monomeric connectin. If one assumes $5 \times 10^{4}$ connectin molecules/cell [3], then the clustering of connectin into a dimer would enhance its affinity for actin by $10^{4}$ compared with monomeric receptor. Clustering of connectin into a tetramer would enhance its actin binding by $10^{12}$ compared with monomer (see $[19,20]$ for the mathematical discussion of interaction of transmembrane proteins with the cytoskeleton). A similar effect of clustering of conA receptors on their association with the cellular cytoskeleton has been reported for lymphocytes [17].

There is now increasing evidence that cells may have specific receptors for extracellular matrix components other than laminin. Both fibronectin and collagen may interact with the cell surface via specific membrane receptors $[9,10]$. Furthermore, there is evidence for a transmembrane association of these extracellular matrix components with the cellular cytoskeleton [21-23]. Findings reported in our studies, taken together with these findings, indicate that the interactions between extracellular matrix and cytoskeleton may be mediated by specific transmembrane extracellular matrix receptors.

We would like to thank Mr George Lowrie and Ms Angela Yang for excellent technical assistance. This work was supported by grants BC357 from the American Cancer Society, HD16721 and GM37091 from the NIH and a grant from the Pardee Foundation.

\section{REFERENCES}

1. Hay, E D, Cell biology of extracellular matrix. Plenum Press, New York (1981).

2. Bissel, M J, Hall, G \& Parry, G, J theor biol 99 (1982) 31.

3. Malinoff, H L \& Wicha, M W, J cell biol 96 (1983) 1475.

4. Rao, N C, Barsky, S H, Terranova, V P \& Liotta, L A, Biochem biophys res commun 111 (1983) 804 .

5. Lesot, H, Kuhl, U \& Von der Mark, K, Embo j (1983) 861.

6. Huard, T K \& Wicha, M S, Clin res 32 (1984) 349A.

7. Koda, J E \& Bernfield, M, J biol chem 159 (1984) 11763.

8. - Ibid 260 (1985) 8157.

9. Yamada, K, Ann rev biochem 52 (1983) 761.

10. Pytela, R, Pierschbacher, M D \& Ruoslahti, E, Cell 40 (1985) 191.

11. Sugrue, S P \& Hay, E D, J cell biol 91 (1981) 45.

12. Brown, S S, Malinoff, H L \& Wicha, M S, Proc natl acad sci US 80 (1983) 5927.

13. Varani J \& Lovett, E J, J natl cancer inst 68 (1982) 957.

14. Timpl, R, Rohoe, H, Robey, G, Rennard, S P, Foidart, J M \& Martin, G M, J biol chem 254 (1979) 9933.

15. Wicha, M S \& Huard, T K, Exp cell res 143 (1983) 475.

16. Foidart, J M, Berre E W, Yaar, M, Rennard, S I, Gullino, M, Martin, G R \& Katz, S I, Lab invest $42(1980) 336$. 


\section{Cody and Wicha}

17. Bourguignon, L Y W \& Singer, S J, Proc natl acad sci US 74 (1977) 5031.

18. Shibata, S, Peters, B P, Roberts, D D, Goldstein, I J \& Liotta, L A, FEBS lett 142 (1982) 194.

19. Jacobson, B S, Tissue and cell 15 (1983) 829.

20. Brandts, J F \& Jacobson, B S, Surv syn path res 2 (1983) 107.

21. Chen, W, Hasegawa, E, Hasegawa, T, Weinstock, C \& Yamada, K M, J cell biol 100 (1985) 1103.

22. Yurchenco, P D, Tsi Library, E C, Charonis, A S \& Furthmayr, H, J biol chem 260 (1985) 7636.

23. Hamada, H, Sato M, Ozawa, M, Yoshitada, O \& Muramatsu, T, Exp cell res 154 (1984) 299.

Received October 16, 1985

Revised version received December 23, 1985 